\title{
Describing Point of Entry into Care and Being Lost to Program in a Cohort of HIV Positive Pregnant Women in a Large Urban Centre in Uganda
}

\author{
Rachel Musomba, ${ }^{1}$ Frank Mubiru, ${ }^{1}$ Shadia Nakalema, ${ }^{1}$ Hope Mackline, ${ }^{1}$ Ivan Kalule, \\ Agnes N. Kiragga, ${ }^{1}$ Rosalind Parkes Ratanshi, ${ }^{1,2}$ and Barbara Castelnuovo ${ }^{1}$ \\ ${ }^{1}$ Infectious Diseases Institute, Makerere University, Mulago Hospital, Kampala, Uganda \\ ${ }^{2}$ Cambridge Institute of Public Health, Cambridge, UK \\ Correspondence should be addressed to Rachel Musomba; rmusomba@idi.co.ug
}

Received 18 November 2016; Revised 19 January 2017; Accepted 19 February 2017; Published 2 April 2017

Academic Editor: Fengliang Liu

Copyright ( 2017 Rachel Musomba et al. This is an open access article distributed under the Creative Commons Attribution License, which permits unrestricted use, distribution, and reproduction in any medium, provided the original work is properly cited.

\begin{abstract}
Introduction. We aim to describe the time of entry into care and factors associated with being lost to program (LTP) in pregnant women on Option B Plus in an integrated HIV and antenatal care (ANC) clinic in Uganda. Methods. We included all pregnant women enrolled into the integrated HIV-ANC clinic from January 2012 to 31st July 2014, while the follow up period extended up to October 30th 2015. LTP was defined as being out of care for $\geq 3$ months. Results. Overall 856 women were included. Only $36.4 \%$ $(86 / 236)$ of the women were enrolled in the first trimester. Overall $69(8.1 \%)$ were LTP. In the multivariate analysis older women (HR: 0.80 per five-year increase, CI: $0.64-1.0$, and $P=0.060)$ and women on ART at the time of pregnancy $(0.58, \mathrm{CI}: 0.34-0.98$, and $P=0.040$ ) were more likely not to be LTP. Among women already on ART at the time of pregnancy no factor was associated with LTP. Conclusion. Our results suggest the need for interventions to enhance prompt linkage of HIV positive women to HIV services for ART initiation and for increased retention particularly in young and ART naive women.
\end{abstract}

\section{Background}

In many developed countries, paediatric HIV has been virtually eliminated [1]. The United Nations programme on HIV/AIDS (UNAIDS) estimates that currently 330,000 children are infected with HIV/AIDS worldwide, $90 \%$ of whom are living in Sub-Saharan Africa [2,3]. Mother-tochild transmission (MTCT) is the primary mode of HIV infection in children either during pregnancy, labor, and delivery or through breast feeding [4-6]. Several studies have proven that antiretroviral drugs decrease HIV transmission from the mother to the baby [7-11]. Particularly, the use of triple antiretroviral (ART) therapy during pregnancy has been proven to be highly effective in reducing motherto-child HIV transmission from $15 \%$ up to $45 \%$ with no treatment at all to below 5\% [12].
To attain virtual elimination of paediatric HIV, programs in resource limited settings have scaled up integrated prevention of mother-to-child transmission (PMTCT) programs to identify and enroll HIV infected pregnant women into care and treatment services [13-16].

Since 2012, the WHO recommends immediate start of lifelong ART constituted by tenofovir, emtricitabine, and efavirenz, for all HIV positive pregnant and breast feeding women presenting into care regardless of their $\mathrm{CD} 4$ count or WHO clinical staging; this strategy is also known as Option B Plus [17].

Despite programs' efforts to scale up Option B Plus strategy by $50 \%$ coverage [18] and with up to $68.1 \%$ women starting ART during antenatal care and after delivery in SubSaharan Africa, the effectiveness of this prevention strategy to move towards an HIV free generation [19] is undermined 
by high rates of being lost to program (LTP) [20]. The rates of LTP are particularly alarming in the postpartum period (up to $50 \%)[21,22]$.

So far most of the national programs in Sub-Saharan Africa, while challenged with low retention in care of mothers and their infants, are limited with interventions to improve retention into care and reduce LTP [23].

In Uganda, routine HIV screening is recommended for all women presenting for antenatal or delivery care in all public health facilities [24]; women who test HIV positive are offered, in addition to antenatal care (ANC), Option B Plus during prenatal and postnatal visits up to 18 months after delivering and are thereafter referred to HIV care services. However an earlier evaluation from public clinics in Kampala, the capital city, revealed alarming rates of being lost to followup between $25 \%$ and $58.8 \%$ [25].

At the Infectious Diseases Institute (IDI), Kampala, Uganda, an HIV centre of excellence, in order to increase retention into care and to avoid referrals to and from ANC services, with potential loss from care, an integrated HIVANC clinic was implemented in 2012. The main objective of this study was to describe the time of entry into care into the integrated HIV-ANC clinic and investigate factors associated with being lost to program.

\section{Methods}

2.1. Study Setting and Population. The Infectious Diseases Institute (IDI), Makerere University, is an HIV centre of excellence [26] located in Mulago Teaching Hospital in Kampala with over 8,000 HIV positive individuals receiving care. The IDI clinic began providing HIV care in 2002, while free antiretroviral treatment has been provided since April 2004. In 2012 an integrated HIV-ANC clinic was put into service, where all pregnant women receive antenatal and HIV care including Option B Plus.

2.2. The Integrated HIV-ANC Clinic. Women suspected to be pregnant on the basis of the date of their last menstrual period receive a targeted pregnancy confirmatory test, and if found positive they are referred to the integrated HIV-ANC clinic, while new HIV positive pregnant or breast feeding women referred to IDI are directed to the HIV-ANC clinic immediately at enrolment into care. In this clinic, pregnant women are offered iron and folic acid supplementation, antiretroviral drugs, prevention and management of opportunistic infections, education on obstetric practices, especially during labor and delivery, and counseling on infant feeding options. Mothers already on ART and those ART naive are prepared to start or switch to tenofovir, emtricitabine, and efavirenz as part of Option B Plus. At the time of this analysis ART was monitored using CD4 counts; viral load testing was not routinely available.

The clinic is staffed by a trained team of 3 medical officers, 2 nurses, and a nurse-counselor; it is supervised by a senior medical officer and assisted in logistic and health education tasks by a peer supporter.
2.3. Data Collection. At IDI patients' clinical information is captured into an in-house built provider-based electronic medical record EMR system called Integrated Clinic Enterprise Application (ICEA) [27]. The system generates automated queries to eliminate omission of mandatory fields and has internal consistency checks which ensure that the data entered is accurate. Within the main patient's management application, information on pregnant women and their babies can be entered in a dedicated module.

The Option B Plus ICEA module captures data before and after delivery which include information on whether the pregnancy was intended, gravity, parity, number of abortions, last normal menstrual period, expected date of delivery, and birth outcomes.

2.4. Statistical Analysis. In this analysis, we included all pregnant women who started ART in the HIV-ANC integrated clinic from 1st January 2012, the year Option B Plus was implemented, to 31st July 2014, while the follow-up period extended up to 30th October 2015. We used proportion to describe the point of entry into care (1st, 2nd, and 3rd trimester, on the day of delivery and postpartum) and the magnitude and reason of discontinuation from care (LTP, death, or transferred to another program). We used KaplanMeier survival analysis to estimate time to LTP. A woman was defined as LTP if she was alive but had not come back for her appointment for at least 3 months; women who had been transferred out of care but were attending another facility and receiving ART were not considered LTP.

Women were followed up from time of enrolment into the ANC to date of last clinic encounter or date of database closure for those still in care. We used Cox proportional hazards model methods to identify factors associated with LTP among women enrolled into the integrated HIV-ANC. Variables included in the model were age, WHO stage, parity, CD4 count, and ART status (ART naive versus on ART). Variables with a $P$ value $\leq 0.25$ in the unadjusted Cox proportional hazards model and those of clinical significance were included in the multivariate model. We also conducted a subanalysis including women already on ART at the time of pregnancy.

Analysis was performed using STATA 12.2, Texas, USA.

2.5. Ethical Statement. This study was approved and annually renewed by the School of Medicine Research and Ethics Committee, Makerere University Medical School (Reference number 2009-120), and the Uganda National Council for Science and Technology. The investigators obtained verbal or written consent waiver; all the information is analysed after stripping it of unique personal identifiers.

\section{Results}

Overall 856 pregnant women were included in the analysis; at the time of the enrolment into the HIV-ANC integrated clinic, the median age was 31 years (IQR: 26-35), and 302 


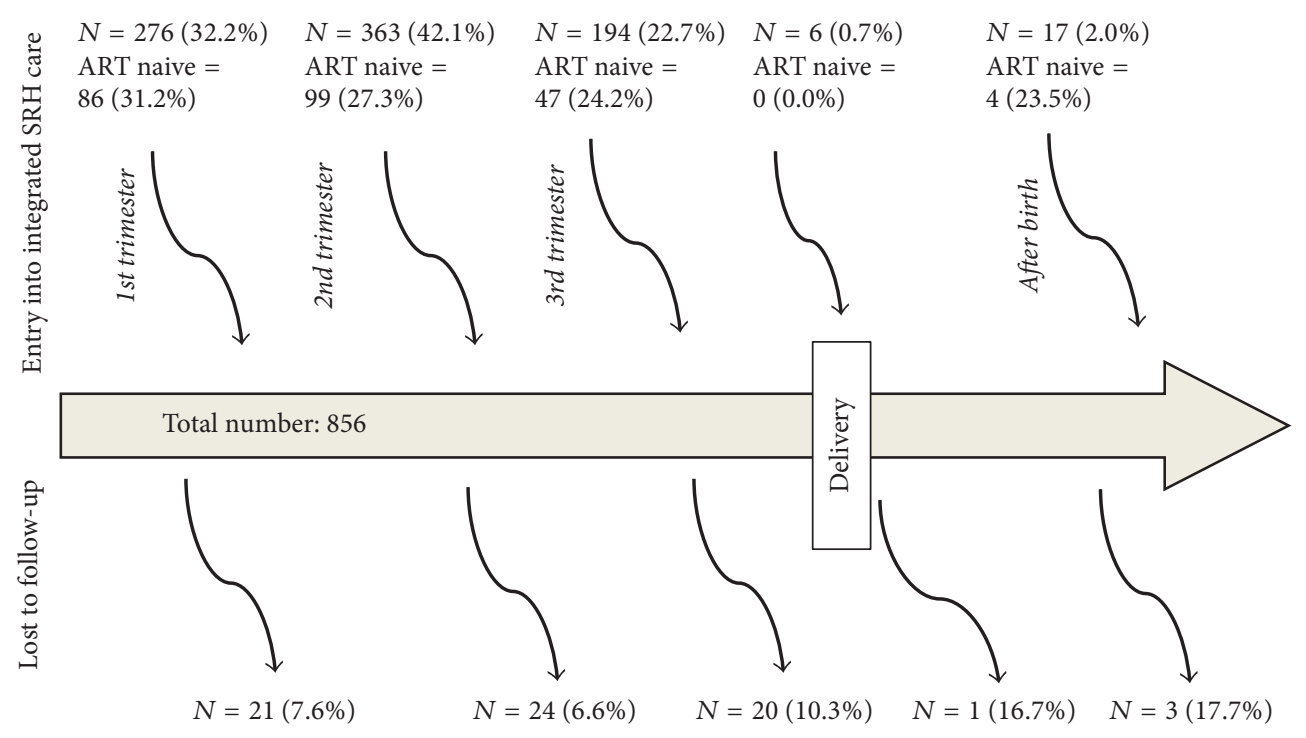

FIGURE 1: Showing point of entry and being lost to program by gestational age.

(35.3\%) were in WHO stage 3/4; the median CD4 count was 433 cells/ $\mu$ L (IQR: 301-638), 236 (27.6\%) were ART naive, and 329 (39.5\%) had already had at least 2 pregnancies (Table 1 ).

Figure 1 shows the time of entry into care in the integrated HIV-ANC clinic by gestational age and numbers of patients LTP. Only $32.2 \%(86 / 276)$ of the women were enrolled in the first trimester, and of these $86(31.2 \%)$ were ART naive. Three hundred and sixty-three women (42.1\%) were enrolled in the 2nd trimester, and of these 99 (27.3\%) were ART naive; only $17(2 \%)$ of patients were enrolled after birth, and among these 4 (23.5\%) were ART naive.

The overall proportion of women LTP was $8.1 \%(69 / 856)$ with higher proportion of LTP observed in the postdelivery period (Figure 1).

There was a total follow-up time of 22262 person months. The median time to loss to follow-up was $12.4(4.2-21.3)$ months and active patients had a follow-up time of 28.8 (20.7-37.5) months.

Figure 2 shows the cumulative probability of being LTFU by ART status; women who were ART naive at the time of pregnancy had a higher cumulative probability $(0.155,95 \%$ CI: $0.102-0.232)$ of being LTFU as compared to those already on ART $(0.085,95 \%$ CI: $0.0587-0.123)(P=0.025)$.

In the univariate analysis older age (HR: 0.77, CI: $0.62-0.96$, and $P=0.019$ ) being on ART at the time of pregnancy (HR: 0.56, CI: $0.33-0.94$, and $P=0.0270$ ) was associated with lower risk of being lost to program (Table 2).

In the multivariate analysis women of older age (HR: 0.80 per five-year increase, CI: 0.64-1.0, and $P=0.060)$ and women on ART at the time of pregnancy (0.58, CI: 0.34-0.98, and $P=0.040)$ remained factors associated with reduced LTFU. In the subanalysis that included only women who were not ART naive at the time of pregnancy, we found no factors associated significantly with LTP.
TABLE 1: Characteristic of pregnant women at enrollment in the integrated HIV-antenatal clinic.

\begin{tabular}{lc}
\hline Characteristics & $N=856$ \\
\hline Age (years), median (IQR) & $31(26-35)$ \\
Stage of pregnancy, $n(\%)$ & \\
$\quad$ First trimester & $276(32.2)$ \\
Second trimester & $363(42.4)$ \\
Third trimester & $194(22.7)$ \\
On delivery & $6(0.7)$ \\
After birth & $17(2.0)$ \\
Already on ART, $n(\%)$ & $620(72.4)$ \\
WHO clinical stage 3/4, $n(\%)$ & $302(35.3)$ \\
Parity $>2, N(\%)$ & $329(39.5)$ \\
CD4 cells/ $\mu \mathrm{L}$, median $(\mathrm{IQR})$ & $433(301-638)$ \\
$n(\%) \leq 350$ & $288(33.7)$ \\
$351-500$ & $219(25.6)$ \\
$>500$ & $347(40.6)$ \\
\hline ART:
\end{tabular}

ART: antiretroviral treatment.

\section{Discussion}

In this study we described the point of entry and being lost to program in an integrated HIV-ANC clinic in Kampala, Uganda. We found that in our program rates of LTP are lower, as compared to other programs in in Sub-Saharan Africa; findings from Ethiopia demonstrated 16.5\% [28] and Malawi $17 \%$ [29] of women LTP. Our results suggest that training dedicated staff and integration of services may lead to good levels of retention into care.

However in our program, similarly to what is reported from other countries [30,31] despite overall good retention, only one third of ART naive women joined in the first trimester, with potential risk of transmission to their infants. 
TABLE 2: Factors associated with loss to program using Cox Proportional Hazards model.

\begin{tabular}{|c|c|c|c|c|}
\hline Characteristics & Unadjusted HR (95\% CI) & $P$ value & Adjusted HR (95\% CI) & $P$ value \\
\hline Age per 5 year increase & $0.77(0.62-0.96)$ & 0.019 & $0.80(0.64-1.00)$ & 0.060 \\
\hline \multicolumn{5}{|l|}{ Gestational age } \\
\hline First trimester & 1.00 & & & \\
\hline Second trimester & $0.75(0.40-1.41)$ & 0.370 & $0.78(0.42-1.46)$ & 0.439 \\
\hline Third trimester & $1.44(0.77-2.69)$ & 0.260 & $1.52(0.80-2.82)$ & 0.210 \\
\hline On delivery & $3.45(0.46-25.81)$ & 0.230 & $4.22(0.56-31.9)$ & 0.163 \\
\hline After birth & $2.00(0.47-8.58)$ & 0.350 & $2.20(0.51-9.42)$ & 0.290 \\
\hline \multicolumn{5}{|l|}{ ART status at enrollment } \\
\hline ART naive & 1.00 & & & \\
\hline On ART & $0.56(0.33-0.94)$ & 0.027 & $0.58(0.34-0.98)$ & 0.040 \\
\hline \multicolumn{5}{|l|}{ WHO clinical stage } \\
\hline I-II & 1.00 & & & \\
\hline III-IV & $0.91(0.54-1.54)$ & 0.730 & & \\
\hline \multicolumn{5}{|l|}{ Parity } \\
\hline$\leq 2$ & 1.00 & & & \\
\hline$>2$ & $0.82(0.48-1.39)$ & 0.454 & & \\
\hline \multicolumn{5}{|l|}{ CD 4 cells $/ \mu \mathrm{L}$} \\
\hline$\leq 350$ & 1.00 & & & \\
\hline $350-500$ & $0.98(0.51-1.92)$ & 0.980 & & \\
\hline$>500$ & $0.97(0.54-1.73)$ & 0.910 & & \\
\hline
\end{tabular}

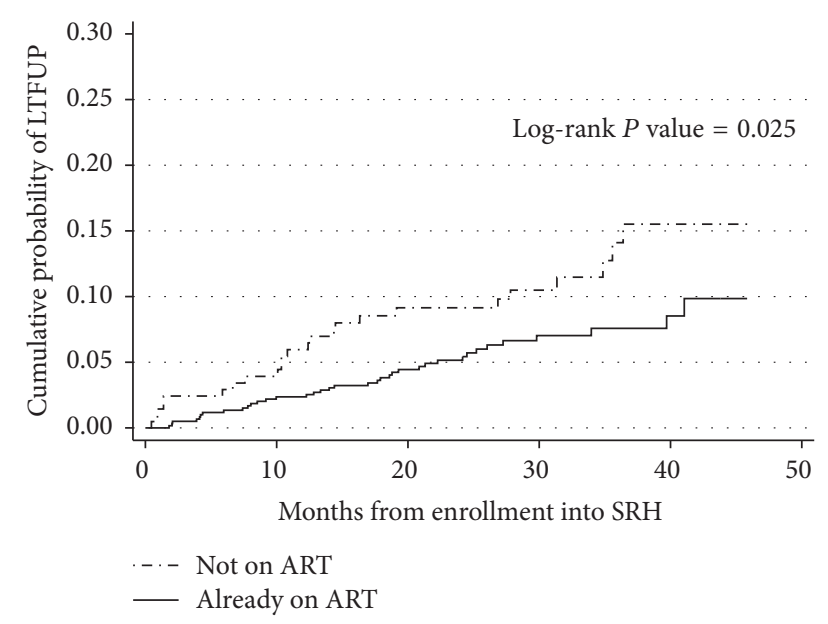

FIGURE 2: Showing cumulative probability of being lost to program by ART status.

In our study we found higher rates of LTP in younger women and in women who were ART naive at the time of pregnancy and in the postpartum period. The high rates of LTP among young women are likely to reflect the low retention into the cascade from testing HIV positive to being uninterruptedly on treatment observed in this group [32]. Several factors may contribute to this; for example, young people are less concerned about their health compared to older people, they tend to have more risky lifestyle due to a youthful sense of invulnerability, and they are usually healthier and often engage into activities that interfere with routine clinic scheduled visits [33-35]. Additionally, stigma related to pregnancy among young people can influence retention in care $[36,37]$.

We also found that women on ART at the time of pregnancy were less likely to be LTP. Our findings are consistent with those of previous studies which indicated that ART experienced patients are more likely to be retained in care [28].

We observed that LTP was higher during postpartum period. Similar findings were reported in a cohort study done in South Africa in which $49 \%$ of women had disengaged from care postpartum [21]. These findings are also consistent with results from other studies [21, 38, 39]. We can hypothesize that, with the decentralization of HIV services in including provision of antiretroviral drugs, a proportion of women selftransfer to facilities nearer to their home after delivery [40]. This is possible in order to reduce transport costs.

Lack of disclosure of HIV positive status has been associated with increased LTFU $[23,41]$ in women enrolled in PMTCT programs. We hypothesize that women who have not disclosed report to their partners that they visit monthly a health care facility for ANC, but not for HIV care; after delivering they may stop coming to the clinic since they cannot justify to their partners' monthly visits to a health care facility.

One limitation of this study was that the information was obtained from routinely collected data, which may pose challenges in terms of data quality. However a previous evaluation of our database showed high completeness and consistency of data [27]. Another limitation is that we did not have preintegrated HIV-ANC clinic data for comparison purposes, and therefore we cannot attribute the high retention demonstrated in this study entirely to HIV-ANC integrated 
clinic, as other measures have been implemented at the entire clinic level to improve retention in for all patients.

Although reports from observation studies in SubSaharan Africa seem to suggest that integration of ANC including Option $\mathrm{B}+$ and HIV services improves retention, this needs to be validated in more rigorous studies [42].

\section{Conclusion}

While in our program we achieved good retention of pregnant women enrolled in the Option $\mathrm{B}+$ program, integration of services may not be sufficient to reduce being lost to program among young women and those who are naive. Where available, for groups at risk of being lost to program, resources should be utilized for other interventions which have demonstrated to be effective in increasing retention.

\section{Disclosure}

This study was self-funded.

\section{Conflicts of Interest}

All authors declare that they have no conflicts of interest.

\section{Authors' Contributions}

Agnes N. Kiragga conceived the study and supervised the data extraction and analysis, Frank Mubiru extracted and analysed the data, Ivan Kalule validated the data for completeness, and Shadia Nakalema and Hope Mackline reviewed the manuscript and are involved in the implementation of the Option B Plus. Barbara Castelnuovo overall supervised the study and reviewed the manuscript. All authors have reviewed and approved the manuscript.

\section{References}

[1] S. O. Mepham, R. M. Bland, and M.-L. Newell, "Prevention of mother-to-child transmission of HIV in resource-rich and poor settings," BJOG: An International Journal of Obstetrics and Gynaecology, vol. 118, no. 2, pp. 202-218, 2011.

[2] M. Ostergren and R. Malyuta, "Elimination of HIV infection in infants in Europe-challenges and demand for response," Seminars in Fetal and Neonatal Medicine, vol. 11, no. 1, pp. 54-57, 2006.

[3] UNAIDS Joint United Nations Programme on HIV/AIDS, "2015 progress report on the global plan towards the elimination of new HIV infections among children and keeping their mothers alive," 2013.

[4] E. Tchiakpe, A. Hounto-Ogouyemi, H. Diop Ndiaye et al., "Use of dried blood spots in early diagnosis of HIV-1 infection in children born to HIV-infected mothers as part of the prevention of mother-to-child transmission in Benin," Bulletin de la Societe de Pathologie Exotique, vol. 109, no. 3, pp. 155-159, 2016.

[5] S. Nesheim, A. Taylor, M. A. Lampe et al., "A framework for elimination of perinatal transmission of HIV in the United States," Pediatrics, vol. 130, no. 4, pp. 738-744, 2012.
[6] G. S. Birkhead, W. P. Pulver, B. L. Warren et al., "Progress in prevention of mother-to-child transmission of HIV in New York state: 1988-2008," Journal of Public Health Management and Practice, vol. 16, no. 6, pp. 481-491, 2010.

[7] Y. A. R. Lima, L. P. V. Cardoso, M. N. D. G. Reis, and M. M. A. Stefani, "Incident and long-term HIV-1 infection among pregnant women in Brazil: transmitted drug resistance and mother-to-child transmission," Journal of Medical Virology, vol. 88, no. 11, pp. 1936-1943, 2016.

[8] F. Wudineh and B. Damtew, "Mother-to-child transmission of HIV infection and its determinants among exposed infants on care and follow-up in Dire Dawa City, Eastern Ethiopia," AIDS Research and Treatment, vol. 2016, Article ID 3262746, 6 pages, 2016.

[9] M. Coulibaly, E. Thio, C. Yonaba et al., "Prevention and care of paediatric HIV infection in Ouagadougou, Burkina Faso: knowledge, attitudes and practices of the caregivers," $B M C$ Pediatrics, vol. 16, no. 1, article 33, 2016.

[10] S. R. Permar, Y. Fong, N. Vandergrift et al., "Maternal HIV1 envelope-specific antibody responses and reduced risk of perinatal transmission," Journal of Clinical Investigation, vol. 125, no. 7, pp. 2702-2706, 2015.

[11] AIDSinfo, Preventing Mother-to-Child Transmission of HIV, 2014.

[12] WHO, "Mother-to-child transmission of HIV report," 2015.

[13] B. H. Chi, C. Bolton-Moore, and C. B. Holmes, "Prevention of mother-to-child HIV transmission within the continuum of maternal, newborn, and child health services," Current Opinion in HIV and AIDS, vol. 8, no. 5, pp. 498-503, 2013.

[14] M. B. Rasmussen, J. B. Rasmussen, V. R. Nielsen et al., "Prevention of vertical transmission of HIV in Denmark," Ugeskrift for Laeger, vol. 170, no. 34, pp. 2567-2570, 2008.

[15] J. Neubert, M. Pfeffer, A. Borkhardt et al., "Risk adapted transmission prophylaxis to prevent vertical HIV-1 transmission: effectiveness and safety of an abbreviated regimen of postnatal oral Zidovudine," BMC Pregnancy and Childbirth, vol. 13, article 22, pp. 1471-2393, 2013.

[16] R. Becquet, D. K. Ekouevi, E. Arrive et al., "Universal antiretroviral therapy for pregnant and breast-feeding HIV-1-infected women: towards the elimination of mother-to-child transmission of HIV-1 in resource-limited settings," Clinical Infectious Diseases, vol. 49, no. 12, pp. 1936-1945, 2009.

[17] WHO, Use of Antiretroviral Drugs for Treating Pregnant Women and Preventing HIV Infection in Infants-Programmatic Update, WHO/HIV/2012.6, WHO, Geneva, Switzerland, 2012.

[18] M. P. Kieffer, M. Mattingly, A. Giphart et al., "Lessons learned from early implementation of option B+: the elizabeth glaser pediatric AIDS foundation experience in 11 African countries," Journal of Acquired Immune Deficiency Syndromes, vol. 67, pp. S188-S194, 2014.

[19] M. Musinguzi and D. 't Hart, Towards an AIDS Free Generation in Uganda, Starting with the Children in Uganda, 2015.

[20] E. L. Sibanda, I. V. D. Weller, J. G. Hakim, and F. M. Cowan, "The magnitude of loss to follow-up of HIV-exposed infants along the prevention of mother-to-child HIV transmission continuum of care: a systematic review and meta-analysis," AIDS, vol. 27, no. 17, pp. 2787-2797, 2013.

[21] T. Phillips, E. Thebus, L.-G. Bekker, J. Mcintyre, E. J. Abrams, and L. Myer, "Disengagement of HIV-positive pregnant and postpartum women from antiretroviral therapy services: a cohort study," Journal of the International AIDS Society, vol. 17, Article ID 19242, 2014. 
[22] E. N. Kurewa, G. Q. Kandawasvika, F. Mhlanga et al., "Realities and challenges of a five year follow up of mother and child pairs on a PMTCT program in Zimbabwe," Open AIDS Journal, vol. 5, no. 1, pp. 51-58, 2011.

[23] R. M. Hoffman, "Disclosure and knowledge are associated with retention in Malawi's option B+ program," in Proceedings of the Conference on Retroviruses and Opportunistic Infections (CROI '16), Boston, Mass, USA, February 2016.

[24] Ministry of Health (MOH), The Integrated National Guidelines on Antiretroviral Therapy, Prevention of Mother to Child Transmission of HIV and Infant \& Young Child Feeding, Uganda, 2011.

[25] A. N. Kiragga, R. Mwonda, E. Nassuna, and J. Kigozi, "High rates of loss to follow up among women on option B+ attending care in urban Kampala, Uganda," WHO Report, 2016, http://www.who.int/hiv/topics/mtct/about/en/.

[26] S. Nwaka, A. Ochem, D. Besson et al., "Analysis of panAfrican Centres of excellence in health innovation highlights opportunities and challenges for local innovation and financing in the continent," BMC International Health and Human Rights, vol. 12, article 11, 2012.

[27] B. Castelnuovo, A. Kiragga, V. Afayo et al., "Implementation of provider-based electronic medical records and improvement of the quality of data in a large HIV program in Sub-Saharan Africa," PLoS ONE, vol. 7, no. 12, Article ID e51631, 2012.

[28] I. Mitiku, M. Arefayne, Y. Mesfin, and M. Gizaw, "Factors associated with loss to follow-up among women in Option $\mathrm{B}+$ PMTCT programme in northeast Ethiopia: a retrospective cohort study," Journal of the International AIDS Society, vol. 19, no. 1, Article ID 20662, 2016.

[29] A. D. Haas, L. Tenthani, M. T. Msukwa et al., "Retention in care during the first 3 years of antiretroviral therapy for women in Malawi's option B+ programme: an observational cohort study," The Lancet HIV, vol. 3, no. 4, pp. el75-e182, 2016.

[30] M. Floridia, C. Pinnetti, M. Ravizza et al., "Rate, predictors, and consequences of late antenatal booking in a national cohort study of pregnant women with HIV in Italy," HIV Clinical Trials, vol. 15, no. 3, pp. 104-115, 2014.

[31] M. D. L. Soares, M. I. C. de Oliveira, V. M. Fonseca, A. D. S. Brito, and K. S. da Silva, "Predictors of unawareness of HIV serostatus among women submitted to the rapid HIV test at admittance for delivery," Ciencia e Saude Coletiva, vol. 18, no. 5, pp. 1313-1320, 2013.

[32] V. Ochieng-Ooko, D. Ochieng, J. E. Sidle et al., "Influence of gender on loss to follow-up in a large HIV treatment programme in western kenya," Bulletin of the World Health Organization, vol. 88, no. 9, pp. 681-688, 2010.

[33] S. Lee, S. H. Lee, S. J. Lee et al., "Predictors of poor retention in care of hiv-infected patients receiving antiretroviral therapy in Korea: five-year hospitalbased retrospective cohort study," Journal of Korean Medical Science, vol. 31, no. 3, pp. 376-381, 2016.

[34] D. Israelski, C. Gore-Felton, R. Power, M. J. Wood, and C. Koopman, "Sociodemographic characteristics associated with medical appointment adherence among HIV-seropositive patients seeking treatment in a county outpatient facility," Preventive Medicine, vol. 33, no. 5, pp. 470-475, 2001.

[35] S. Krishnan, K. Wu, M. Smurzynski et al., "Incidence rate of and factors associated with loss to follow-up in a longitudinal cohort of antiretroviral-treated HIV-infected persons: an AIDS clinical trials group (ACTG) longitudinal linked randomized trials (ALLRT) analysis," HIV Clinical Trials, vol. 12, no. 4, pp. 190-200, 2011.
[36] L. Pretorius, A. Gibbs, T. Crankshaw, and S. Willan, "Interventions targeting sexual and reproductive health and rights outcomes of young people living with HIV: a comprehensive review of current interventions from sub-Saharan Africa," Global Health Action, vol. 8, no. 1, Article ID 28454, 2015.

[37] C. Varga and H. Brookes, "Factors influencing teen mothers' enrollment and participation in prevention of mother-to-child HIV transmission services in limpopo province, South Africa," Qualitative Health Research, vol. 18, no. 6, pp. 786-802, 2008.

[38] P. Ayuo, B. Musick, H. Liu et al., "Frequency and factors associated with adherence to and completion of combination antiretroviral therapy for prevention of mother to child transmission in western Kenya," Journal of the International AIDS Society, vol. 16, Article ID 17994, 2013.

[39] K. Clouse, A. Pettifor, K. Shearer et al., "Loss to follow-up before and after delivery among women testing HIV positive during pregnancy in Johannesburg, South Africa," Tropical Medicine and International Health, vol. 18, no. 4, pp. 451-460, 2013.

[40] E. H. Geng, T. A. Odeny, R. Lyamuya et al., "Retention in care and patient-reported reasons for undocumented transfer or stopping care among HIV-infected patients on antiretroviral therapy in Eastern Africa: application of a sampling-based approach," Clinical Infectious Diseases, vol. 62, no. 7, pp. 935944, 2016.

[41] F. M. Kiweewa, P. M. Bakaki, M. S. McConnell et al., "A crosssectional study of the magnitude, barriers, and outcomes of HIV status disclosure among women participating in a perinatal HIV transmission study, "the Nevirapine Repeat Pregnancy study", BMC Public Health, vol. 15, no. 1, article 988, 2015.

[42] P. Geldsetzer, H. M. N. Yapa, M. Vaikath et al., "A systematic review of interventions to improve postpartum retention of women in PMTCT and ART care," Journal of the International AIDS Society, vol. 19, no. 1, Article ID 20679, 2016. 




The Scientific World Journal
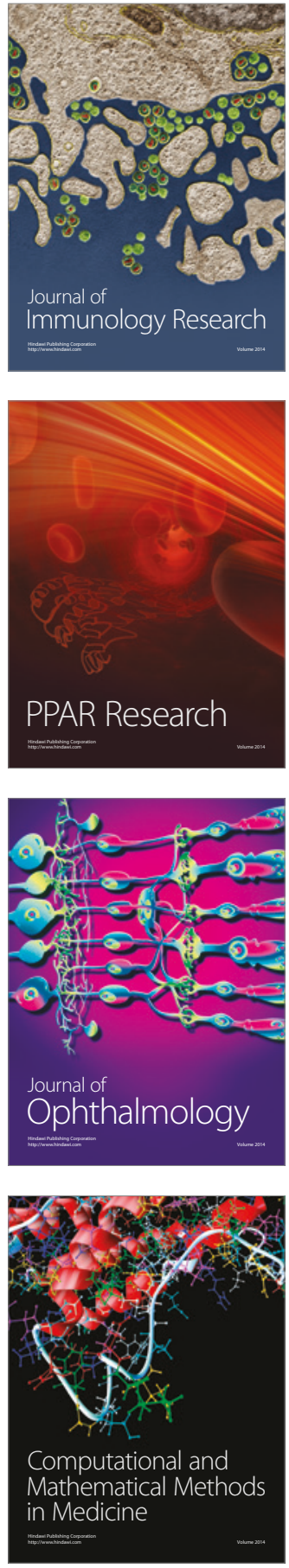

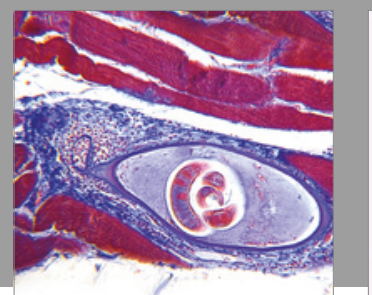

Gastroenterology Research and Practice


\section{Hindawi}

Submit your manuscripts at

https://www.hindawi.com
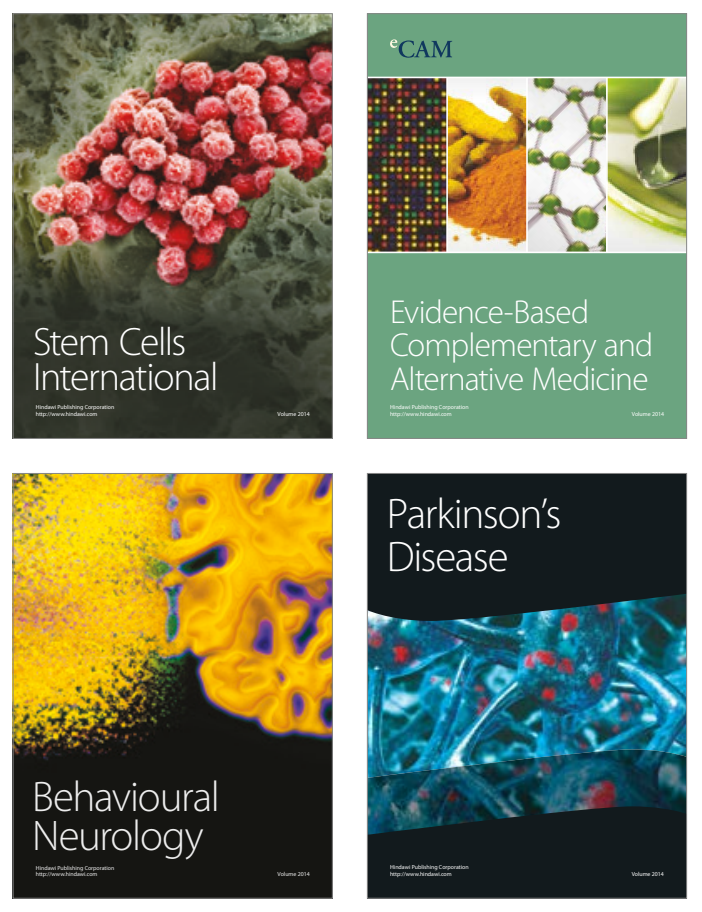
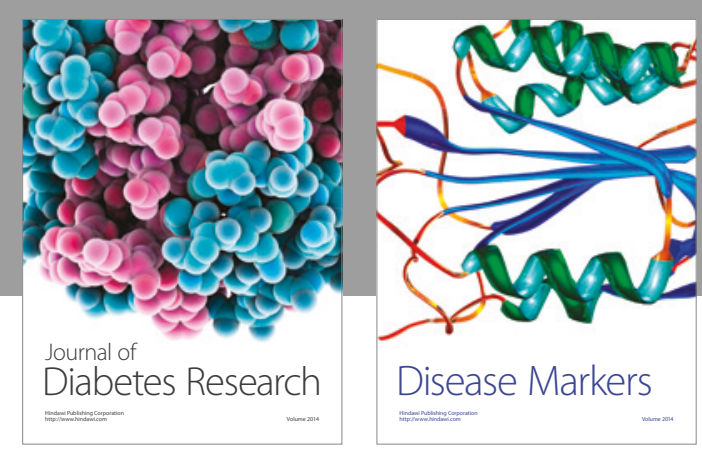

Disease Markers
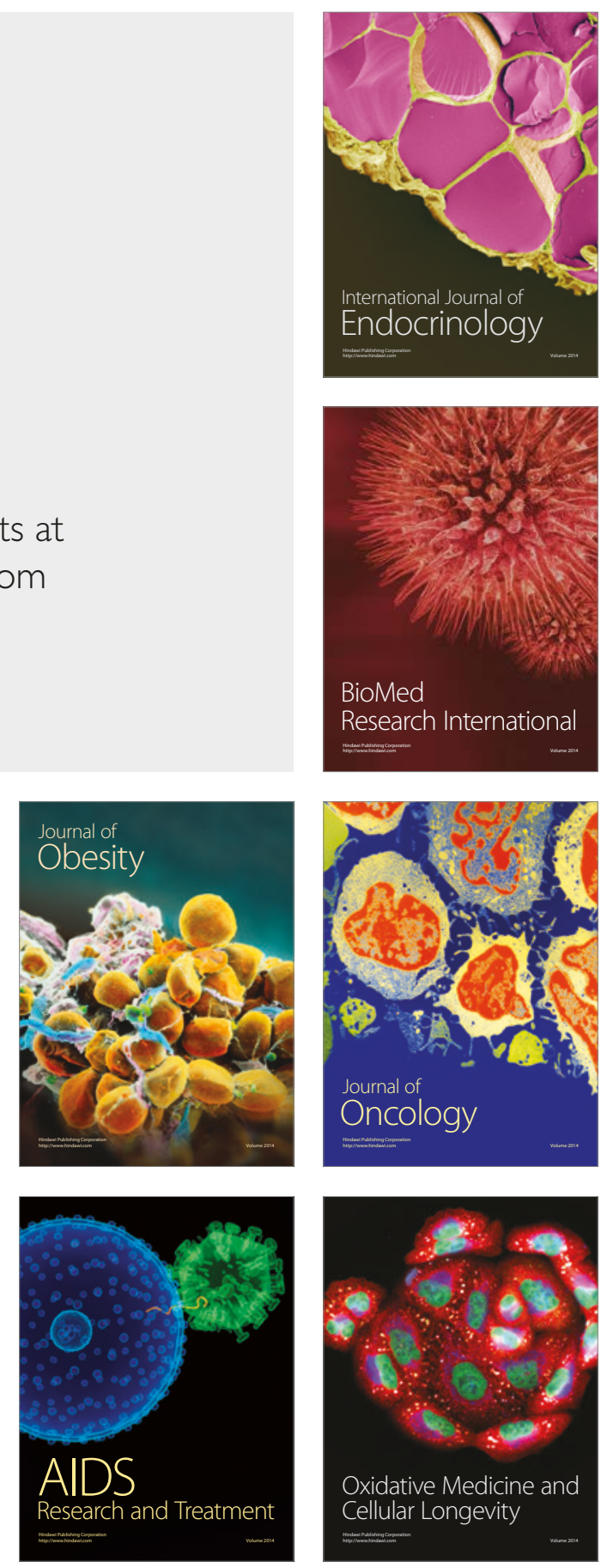